Tim Laux*

\title{
Gradient-flow techniques for the analysis of numerical schemes for multi-phase mean-curvature flow
}

https://doi.org/10.1515/geofl-2018-0006

Received September 15, 2017; accepted November 2, 2017

Abstract: Several recent convergence results for numerical schemes for mean-curvature flow in particular in the multi-phase case with arbitrary surface tensions are discussed. The guiding principle of all these works is the gradient-flow structure of multi-phase mean-curvature flow which is explained in the general framework. For simplicity, the convergence results are presented in the simpler two-phase case.

Keywords: Mean curvature flow, thresholding, MBO scheme, diffusion generated motion, Allen-Cahn equation, multi-phase systems, mean curvature flow

MSC: 35A15, 35K57, 35K93, 74N20

\section{Introduction}

This review article is intended to give an overview over the recent progress in the analysis of numerical schemes for multi-phase mean-curvature flow covering thresholding schemes $[19,20,22]$ and the Allen-Cahn equation [21]. The guiding principle of these proofs is the (degenerate) gradient-flow structure of multi-phase mean-curvature flow, which is explained in detail here. Furthermore, the compatibility of the schemes with this structure is discussed, which is the basis of the convergence proofs. The details of the proofs are not repeated here but the conceptional difficulties for the individual proofs are highlighted and compared.

Phase-field models such as the Allen-Cahn equation

$$
\partial_{t} u_{\varepsilon}=\Delta u_{\varepsilon}-\frac{1}{\varepsilon^{2}} u_{\varepsilon}\left(u_{\varepsilon}^{2}-1\right),
$$

cf. [1], see also (10) below for a more general form, are efficient and versatile computational schemes for mean curvature flow and other geometric evolution equations. A standard method of implementing the Allen-Cahn equation is an operator splitting, alternating between linear diffusion and fast reaction. The diffusion step can be solved explicitly on a regular grid by Fast Fourier Transformation, while the reaction step boils down to solving an ordinary differential equation at each grid point.

The key observation of Merriman, Bence and Osher [27] is that if the time scale $h>0$ in the operator splitting of the scalar Allen-Cahn equation (1) is large compared to the time scale induced by the length scale $\varepsilon$, one does not need to solve the ODE but can replace it by its long-time asymptotics, which results in simple thresholding. The original algorithm can be formulated as follows where $\Omega_{n}$ corresponds to the set $\left\{x: u_{\varepsilon}(x, n h) \approx 1\right\}$.

Algorithm 0.1. Given a time step size $h>0$ and the phase $\Omega_{n-1}$ at time $t=(n-1) h$, obtain the phase $\Omega_{n}$ at time $t=n h$ by:

${ }^{\star}$ Corresponding Author: Tim Laux: Department of Mathematics, University of California, Berkeley, CA 94720-3840 USA, E-mail: tim.laux@berkeley.edu 
1. Convolution step: $\phi_{n}:=G_{h}{ }^{\star} \mathbf{1}_{\Omega_{n-1}}$.

2. Thresholding step: $\Omega_{n}:=\left\{\phi_{n}>\frac{1}{2}\right\}$.

Here and throughout $G_{h}$, denotes the heat kernel at time $h$, i.e., a Gaussian of variance $2 h$, and $\mathbf{1}_{\Omega}$ denotes the characteristic function of the set $\Omega$ taking the value 1 inside $\Omega$ and 0 otherwise. While their idea facilitates the implementation, it causes new difficulties in the analysis as the thresholding map is clearly not a continuous operator on $L^{2}$.

The paper is organized as follows. Section 1 provides some background on literature and a basic introduction to multi-phase mean-curvature flow explaining the (degenerate) gradient-flow structure of multi-phase mean-curvature flow in a mathematically informal but basic and hopefully accessible level. Section 2 deals with the connection between the numerical schemes and the gradient-flow structure, and discusses a series of recent convergence results in collaboration with Felix Otto, Thilo Simon and Drew Swartz at the simpler example of two-phase mean-curvature flow. Section 3 gives an insight into the proofs behind these results and can be used as a manual to read the papers [19-22]. Some open problems are shown in Section 4.

\section{Multi-phase mean-curvature flow as a gradient flow}

One of the most important applications of - and in fact one of the earliest motivations for - mean curvature flow is grain growth, the slow relaxation of grain boundaries in polycrystals [31]. The free energy density $\sigma=\sigma_{i j}(v)$ and the mobility $\mu=\mu_{i j}(v)$ associated to a grain boundary depend on the mismatch of the two adjacent crystal lattices (where the grains are indexed by $i$ and $j$ ) and on the orientation of the grain boundary w.r.t. these lattices, i.e., on the normal $v$ of the interface.

The results presented here apply to a wide class of surface tensions $\sigma_{i j}$, including the most popular ansatz for small angle grain boundaries [32] and might be extended to smooth anisotropies in the future. In the isotropic case with arbitrary surface tensions, the total free energy is given by

$$
\sum_{i, j} \sigma_{i j} \operatorname{Area}\left(\Sigma_{i j}\right),
$$

where $\Sigma_{i j}=\partial \Omega_{i} \cap \partial \Omega_{j}$ denotes the interface between Phases $i$ and $j$, cf. Fig. 2.

Here and throughout, the matrix of surface tensions is assumed to satisfy - next to the obvious symmetry and positivity conditions

$$
\sigma_{i j}=\sigma_{j i}>0 \quad \text { for } i \neq j
$$

and the convention $\sigma_{i i}=0$ - the important triangle inequality

$$
\sigma_{i j} \leq \sigma_{i k}+\sigma_{k j} \text { for any pairwise different indices } i, j, k \text {, }
$$

which guarantees the lower semi-continuity of the energy (2) w.r.t. the convergence of the phases $\Omega_{i}$ in measure. The motion law under consideration here is multi-phase mean-curvature flow, which comes as a system
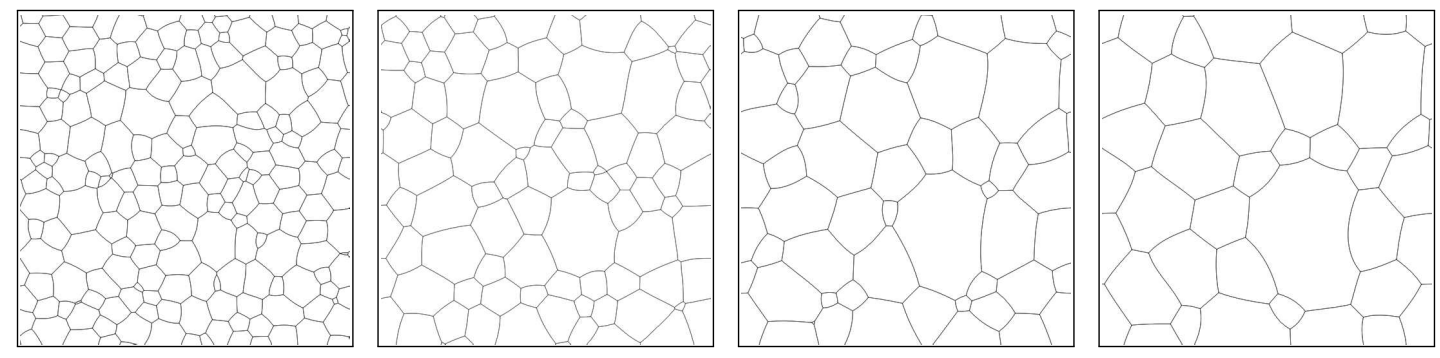

Figure 1: The evolution of a grain boundary network. Computation carried out with the code provided by Selim Esedoğlu [9]. 


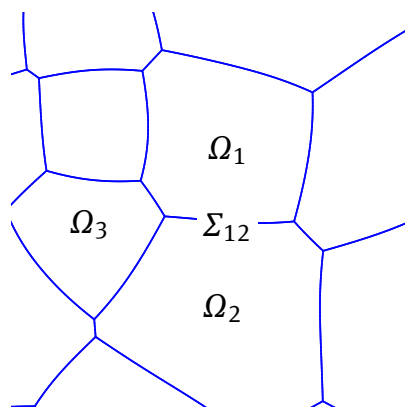

Figure 2: The phases $\Omega_{i}$ and the interfaces $\Sigma_{i j}$ separating them.

of equations of the form

$$
V_{i j}=-\sigma_{i j} \mu_{i j} H_{i j},
$$

where $V_{i j}$ denotes the normal velocity and $H_{i j}$ the mean curvature of the interface $\Sigma_{i j}$; these equations are coupled by the balance of forces condition

$$
\sum_{i, j} \sigma_{i j} v_{i j}=0
$$

along triple junctions, where $v_{i j}$ denotes the normal to the respective interfaces coming together along the junction; in other words, triple junctions are in local equilibrium. An example of such an evolution is given in Figure 1.

Two-phase mean-curvature flow exhibits a geometric comparison principle, which may be formulated as follows: If $\Omega(0)$ and $\widetilde{\Omega}(0)$ are nested, and both move by their mean curvature, then they stay nested, cf. Fig. 3. The same comparison principle holds for thresholding since both operations, convolution with a nonnegative kernel and thresholding, are monotone operators.

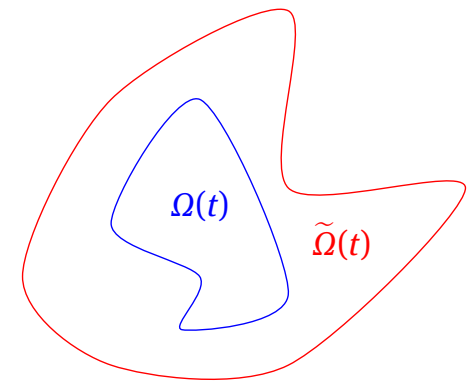

Figure 3: Illustration of the comparison principle.

This comparison principle allows to work in the realm of viscosity solutions, which does not only provide a framework to prove well-posedness despite the non-uniqueness of mean curvature flow which manifests in "fattening" on the level of the viscosity solution [7, 13]. It also gives a straightforward definition of generic flows [14] and its robustness facilitates the development of rigorous long-time convergence results for the two-phase versions of thresholding [5, 11] and the Allen-Cahn equation [12]. However, this notion cannot be generalized to multi-phase mean-curvature flow, where a comparison principle is clearly absent.

Instead, the guiding principle in the work presented here is the gradient-flow structure of (multi-phase) mean-curvature flow. In general, a gradient-flow structure is given by an energy functional and a dissipation mechanism; in the smooth case the dissipation comes from a Riemannian metric on the configuration space. The following simple computation reveals this structure for mean curvature flow: If the network of hypersurfaces $\Sigma_{i j}=\Sigma_{i j}(t)$ evolves smoothly by mean curvature in the sense that all interfaces are smooth, satisfy (4) 
and meet only along triple junctions with the balance of forces condition (5), the change of area is given by

$$
\frac{d}{d t} \sum_{i \neq j} \sigma_{i j} \operatorname{Area}\left(\Sigma_{i j}\right)=\sum_{i \neq j} \sigma_{i j} \int_{\Sigma_{i j}} V_{i j} H_{i j}=-\sum_{i \neq j} \frac{1}{\mu_{i j}} \int_{\Sigma_{i j}} V_{i j}^{2} .
$$

Hence when fixing the energy to be the surface area, the metric tensor is given by the $L^{2}$-metric

$$
\sum_{i \neq j} \frac{1}{\mu_{i j}} \int_{\Sigma_{i j}} V_{i j}^{2}
$$

on the space vector fields that preserve the topology of the network and whose normal component on $\Sigma_{i j}$ is $V_{i j}$. In view of the antisymmetry of the velocities $V_{i j}=-V_{j i}$, this motivates the (negative) definiteness condition on the mobilities

$$
\sum_{i \neq j} \xi_{i} \frac{1}{\mu_{i j}} \xi_{j}<0 \quad \text { whenever } \quad \sum_{i} \xi_{i}=0
$$

which has been discussed by Esedoğlu and Otto [10] at the example of the natural mobilities $\mu_{i j}=\frac{1}{\sigma_{i j}}$ for the thresholding scheme and formally guarantees the non-degeneracy of the metric tensor.

However, it is well-known that even under the condition (7), and even in the simpler two-phase case, some care needs to be taken when dealing with this metric as it is completely degenerate: the geodesic distance vanishes identically [29]. Using the proxy $2 h \int_{\Omega \Delta \Omega_{n-1}} \operatorname{dist}\left(x, \partial \Omega_{n-1}\right) d x$ for the induced distance, Almgren, Taylor and Wang [2] proposed a very influential implicit time discretization for mean curvature flow, which was studied independently by Luckhaus and Sturzenhecker [24] at the same time. Chambolle [6] showed that the scheme [2] can be implemented rather efficiently and simplified the convergence proof to the viscosity solution in [2] significantly. The scheme of Almgren, Taylor and Wang inspired De Giorgi to define a general implicit time discretization for abstract gradient flows which he named "movimenti minimizzanti" or minimizing movements [8] and initiated the still flourishing research on gradient flows on metric spaces, see [3].

Although already Mullins [31] considered mean-curvature flow in order to understand grain boundaries, i.e., multi-phase mean-curvature flow as described above, the mathematical study of these multi-phase systems is fairly young compared to their two-phase counterpart. The analysis of the planar case was initiated by Mantegazza, Novaga and Tortorelli [26] who studied the evolution of a single triple junction with equal surface tensions and in particular generalized Huisken's monotonicity formula [15] to this case. Recently Mantegazza, Novaga and Pluda [25] extended these results to the case of two triple junctions. As in the two-phase case, the monotonicity formula allows to characterize blow-ups at singularities as homethetically shrinking solutions, which have been studied e.g. by Schnürer et al. [36] and in the above mentioned work $[25,26]$. One main difficulty in these works goes under the name "multiplicity-one conjecture", namely that the blow-ups of the solution have unit density. Ilmanen, Neves and Schulze [17] proved short-time existence even when starting from certain non-regular networks by explicit constructions close to homothetically expanding solutions. The recent and substantial existence result of Kim and Tonegawa [18] proves the convergence of (a variant of) Brakke's original scheme to a non-trivial Brakke flow in any space dimension. While in the two-phase case, generic uniqueness follows immediately from the coarea formula applied to the viscosity solution, it is still not clear in the multi-phase case.

The works [19, 21, 22] establish convergence towards a distributional formulation of multi-phase mean curvature flow in the setting of finite perimeter sets introduced by Luckhaus and Sturzenhecker [24] in the twophase case for which the normal velocities can be defined straightforwardly by the distributional equation $\partial_{t} \chi_{i}=V_{i}\left|\nabla \chi_{i}\right|$, where $\chi_{i}$ denotes the characteristic function of the $i$-th phase $\Omega_{i}$. Then one can formulate (4) together with (5) in the following equation

$$
\sum_{i \neq j} \int_{0}^{T} \int_{\Sigma_{i j}}\left(\frac{1}{\mu_{i j}} V_{i} \xi \cdot v_{i}-\sigma_{i j}\left(\nabla \cdot \xi-v_{i} \cdot \nabla \xi v_{i}\right)\right)=0
$$


for any smooth vector field $\xi=\xi(x, t)$. Note that here $V_{i}$ is the normal velocity of the boundary of the phase $\Omega_{i}$ with the sign convention $V_{i}>0$ if $\Omega_{i}$, and in general the interfaces have to be defined via the reduced boundaries $\partial^{\star} \Omega_{i}(t)$ of the time slices, i.e., $\Sigma_{i j}=\Sigma_{i j}(t)=\partial^{\star} \Omega_{i}(t) \cap \partial^{\star} \Omega_{j}(t)$. Furthermore, $v_{i}=v_{i}(x, t)$ denotes the inward unit normal vector to $\partial^{*} \Omega_{i}(t)$, given by the Radon-Nykodym derivative of $\nabla \chi_{i}$ w.r.t. its total variation $\left|\nabla \chi_{i}\right|$. In view of the integration by parts rule

$$
\int_{\Sigma}(\nabla \cdot \xi-v \cdot \nabla \xi v)=\int_{\Gamma} b \cdot \xi-\int_{\Sigma} H \xi \cdot v
$$

for a smooth surface $\Sigma$ with boundary $\Gamma$ and according binormal vector $b$, the curvature-term in the weak formulation encodes the right-hand side of Equation (4) on the smooth part of the interface but also the balance of forces condition (5) along triple junctions. In other words, this distributional formulation is consistent in the sense that if a weak solution is regular (sufficiently smooth, with triple junctions in local equilibrium and without any higher order junctions) it is a classical solution of (4) and (5).

It is easy to see that this notion is stable under strict convergence in $B V$ together with the natural a priori estimate

$$
\sup _{t} \sum_{i \neq j} \sigma_{i j} \operatorname{Area}\left(\Sigma_{i j}\right)+\sum_{i \neq j} \frac{1}{\mu_{i j}} \int_{0}^{T} \int_{\Sigma_{i j}} V_{i}^{2} \leq E_{0} .
$$

The work [20] establishes convergence to another weak formulation of (multi-phase) mean-curvature flow, a special case of Brakke varifold solutions, which take the optimal rate of energy dissipation as the very definition of a weak mean curvature flow. As the metric is completely degenerate, Brakke measures this rate only in terms of the squared mean curvature, not the normal velocity but asks for a family of localized inequalities.

\section{Convergence results}

For applications like grain growth but also in image segmentation it is desirable to design algorithms that are efficient enough to handle large numbers of phases but flexible enough to incorporate external forces, variable surface tensions and even anisotropies.

For phase-field models, the extension to multi-phase systems with arbitrary surface tensions is straightforward: One way to generalize the Allen-Cahn equation to the multi-phase case is to consider the system

$$
\partial_{t} u_{\varepsilon}=\Delta u_{\varepsilon}-\frac{1}{\varepsilon^{2}} \partial_{u} W\left(u_{\varepsilon}\right)
$$

where the order parameter $u_{\varepsilon}: \mathbb{R}^{d} \rightarrow \mathbb{R}^{N}$ is vector valued and the potential $W: \mathbb{R}^{N} \rightarrow[0, \infty)$ has finitely many wells of equal depth. The surface tensions are then given by the geodesic distances of the wells w.r.t. a metric induced by the potential $W$, see (22). Also in this more general case, the equation is a gradient flow for the Cahn-Hilliard energy

$$
F_{\varepsilon}(u)=\int \frac{\varepsilon}{2}|\nabla u|^{2}+\frac{1}{\varepsilon} W(u) d x
$$

which can be seen at the optimal rate of dissipation of energy

$$
\frac{d}{d t} F_{\varepsilon}\left(u_{\varepsilon}\right)=-\varepsilon \int\left|\partial_{t} u_{\varepsilon}\right|^{2} d x
$$

The $\Gamma$-convergence of the functionals $F_{\varepsilon}$ to the total interfacial area was proven by Baldo [4].

Surprisingly, it took more than twenty years to find a similar generalization of the thresholding scheme to the case of arbitrary surface tensions. While the generalization to the multi-phase case with equal surface tensions

$$
\Omega_{n, i}=\left\{G_{h} \star \mathbf{1}_{\Omega_{n-1, i}}>G_{h} \star \mathbf{1}_{\Omega_{n-1, j}} \text { for all } j \neq i\right\}
$$



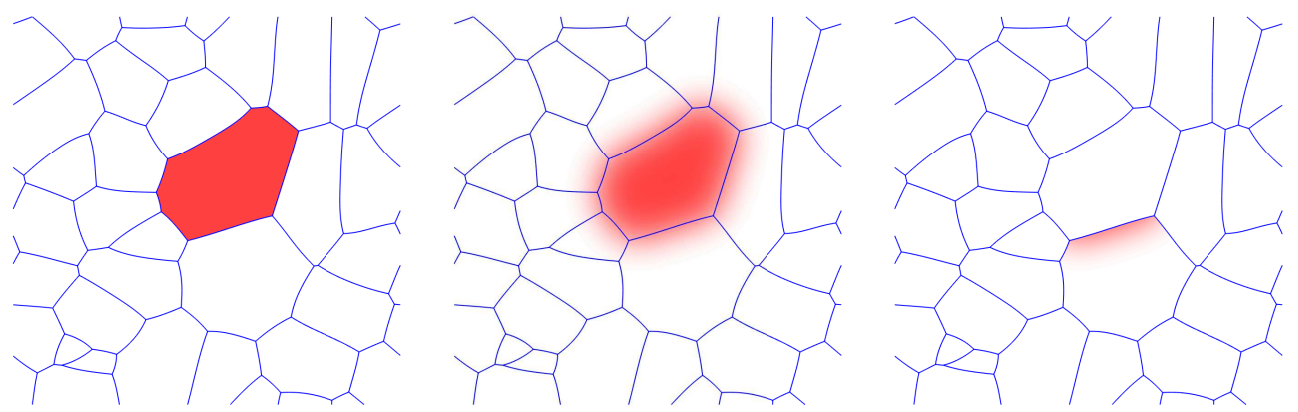

Figure 4: Each summand in the definition of the approximate energy $E_{h}$ is constructed as follows. The phase's characteristic function $\chi_{i}$ (left) gets diffused, yielding $G_{h}{ }^{\star} \chi_{i}$ (center), which is then tested against another characteristic function $\chi_{j}$ (right).

was known for a long time [28], it has not been generalized to the case of different surface tensions until the recent impulse of Esedoğlu and Otto [10] which is inspired by the gradient flow structure of multi-phase mean-curvature flow: The combination of linear diffusion and thresholding is equivalent to

$$
\chi_{n} \in \arg \min \left\{E_{h}(\chi)-E_{h}\left(\chi-\chi_{n-1}\right)\right\},
$$

where the functional $E_{h}$ is given by

$$
E_{h}(\chi)=\frac{1}{\sqrt{h}} \sum_{i \neq j} \int \chi_{i} G_{h}{ }^{\star} \chi_{j} d x
$$

and the minimum runs over all measurable partitions, i.e., $\chi=\left(\chi_{1}, \ldots, \chi_{P}\right)$ such that $\chi_{i} \in\{0,1\}$ and $\sum_{i} \chi_{i}=1$ a.e., cf. [10]. The functionals $E_{h}$ approximate the total interfacial area, see Figure 4. Esedoğlu and Otto found a simple but intriguing proof for the $\Gamma$-convergence of the energies $E_{h}$, which extends to the multi-phase case and follows from the consistency

$$
\lim _{h \rightarrow 0} E_{h}(\chi)=c_{0} \int|\nabla \chi|,
$$

where $c_{0}=\frac{1}{\sqrt{\pi}}$, and the following discrete monotonicity property of the functionals

$$
E_{N^{2} h}(\chi) \leq E_{h}(\chi) \text { for all } h>0, N \in \mathbb{N} .
$$

The second term in the variational problem (12) serves as a proxy of the induced distance, similar to the case of $[2,24]$. Asymptotic expansions for parallel half spaces show that both terms behave equally to first order [10].

The interpretation of the scheme as minimizing movements has several merits. Firstly, by the now obvious choice

$$
E_{h}(\chi)=\frac{1}{\sqrt{h}} \sum_{i \neq j} \sigma_{i j} \int \chi_{i} G_{h}{ }^{\star} \chi_{j} d x,
$$

these functionals approximate the weighted sum (2) of the interfacial energies; it turns out that the thresholding scheme corresponding to the minimization problem (12) with the new functional (15) generalizes the scheme to arbitrary surface tensions with the natural mobilities $\mu_{i j}=\frac{1}{\sigma_{i j}}$ while keeping the same complexity as the scheme with equal surface tensions.

Secondly, this interpretation of the scheme shows that thresholding is compatible with the gradient-flow structure of mean curvature flow and motivated the rigorous convergence analysis [19, 20, 22] and in fact also indirectly the analysis of the Allen-Cahn equation [21].

The following statements hold in the general multi-phase case. However, for the sake of simplicity the results are only stated in the simpler two-phase case. The interested reader is referred to the according cited works for the general statements.

The first theorem is a compactness statement. 
Theorem 2.1 ([19]). Let $T<\infty$ be a finite time horizon and let $\chi_{0}$ be given with $\int\left|\nabla \chi_{0}\right|<\infty$. Then for every sequence $h \rightarrow 0$ there exists a subsequence (which we do not relabel) and $\chi \in L^{\infty}\left((0, T) ; B V\left([0, \Lambda)^{d} ;\{0,1\}\right)\right.$ ) such that the approximate solutions $\chi_{h}$ obtained by Algorithm 0.1 converge to $\chi$.

If the convergence is strong, then the limit is a $B V$-solution.

Theorem 2.2 ([19]). In the situation of Theorem 2.1 if additionally (23) holds, $\chi$ moves by mean curvature in the distributional sense of Definition 2.3.

Definition 2.3. Let $T<\infty$. A measurable time dependent characteristic function $\chi:[0, \Lambda)^{d} \times(0, T) \rightarrow\{0,1\}$ with

$$
\underset{0<t<T}{\operatorname{ess} \sup } \int|\nabla \chi(t)|<\infty
$$

is said to evolve by its mean curvature if there exists a $|\nabla \chi|$-measurable function $V:[0, \Lambda)^{d} \times(0, T) \rightarrow \mathbb{R}$ with

$$
\int_{0}^{T} \int^{2}|\nabla \chi|<\infty
$$

such that $V=-H$ in the sense that

$$
\int_{0}^{T} \int(V \xi \cdot v-(\nabla \cdot \xi-v \cdot \nabla \xi v))|\nabla \chi| d t=0
$$

for all $\xi \in C_{0}^{\infty}\left((0, T) \times[0, \Lambda)^{d}, \mathbb{R}^{d}\right)$ and which are normal velocities in the sense that

$$
\int_{0}^{T} \int \partial_{t} \zeta \chi d x d t=-\int_{0}^{T} \int \zeta V|\nabla \chi| d t
$$

for all $\zeta \in C_{0}^{\infty}\left((0, T) \times[0, \Lambda)^{d}\right)$.

Furthermore, under the same assumption, the limit is a unit-density Brakke flow.

Theorem 2.4 ([20]). In the situation of Theorem 2.1 if additionally (23) holds, then there exists $a|\nabla \chi|^{-}$ measurable function $H$ such that

$$
\int_{0}^{T} \int(\nabla \cdot \xi-v \cdot \nabla \xi v)|\nabla \chi|=-\int_{0}^{T} \int H \xi \cdot v|\nabla \chi|
$$

for all smooth test vector fields $\xi$, and for any smooth and compactly supported test function $\zeta \geq 0$ it holds

$$
\int_{0}^{T} \int\left(\partial_{t} \zeta-\zeta H^{2}+H \nu \cdot \nabla \zeta\right)|\nabla \chi| \geq 0 .
$$

Ruuth and Wetton [34] proposed to change the threshold value from $\frac{1}{2}$ to the number $\lambda=\lambda_{n}$ such that the volume of the phase stays preserved, i.e., the convolution step $\phi_{n}:=G_{h}{ }^{\star} \chi_{n-1}$ is followed by finding the correct value for $\lambda$ such that the according super level set has the same volume, and the final step is the update for the phase

$$
\Omega_{n}=\left\{\phi_{n}>\lambda_{n}\right\} .
$$

The following theorem proves that the limit of this evolution is volume-preserving mean-curvature flow

$$
V=-H+\langle H\rangle,
$$



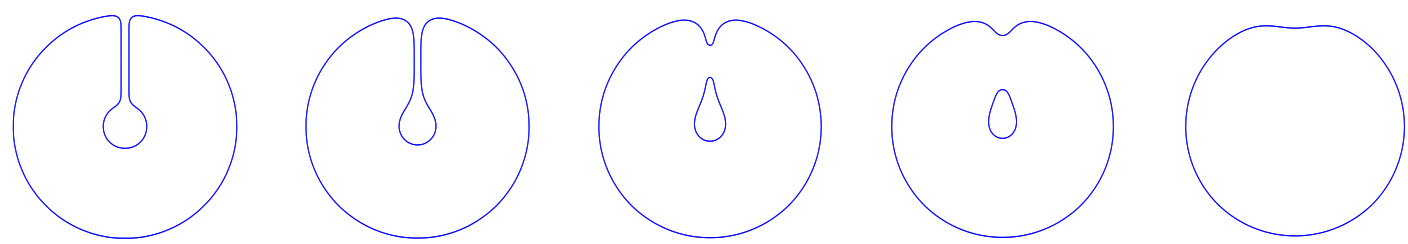

Figure 5: The evolution of a "Thüringer Bratwurst". Computation based on a variant of the code provided by Esedoğlu [9]. The threshold value was computed exactly by sorting the grid points according to their $\phi$-values.

where $\langle H\rangle=\frac{1}{\operatorname{Area}(\Sigma)} \int_{\Sigma} H$ denotes the average of the mean curvature.

As the name suggests, this evolution preserves the enclosed volume and still follows the steepest descent for the area functional w.r.t. the $L^{2}$-metric.

$$
\frac{d}{d t}|\Omega|=\int_{\Sigma} V=0 \text { and } \frac{d}{d t} \operatorname{Area}(\Sigma)=\int_{\Sigma} V H=-\int_{\Sigma} V^{2} .
$$

However, even the evolution of simple planar curves is non-trivial as one can see at Fig. 5.

Theorem 2.5 ([22]). Let $T<\infty$ be a finite time horizon and let $\chi_{0}$ be given with $\int\left|\nabla \chi_{0}\right|<\infty$. Then for every sequence $h \rightarrow 0$ there exists a subsequence (which we do not relabel) and $\chi \in L^{\infty}\left((0, T) ; B V\left(\mathbb{R}^{d} ;\{0,1\}\right)\right.$ ) such that the approximate solutions $\chi_{h}$ obtained by Algorithm 0.1 converge to $\chi$. Furthermore, if additionally (23) holds, then the limit evolves by volume-preserving mean-curvature flow in the sense of Definition 2.3 with (16) replaced by

$$
\int_{0}^{T} \int((V-\Lambda) \xi \cdot v-(\nabla \cdot \xi-v \cdot \nabla \xi v))|\nabla \chi|=0,
$$

where the Lagrange multiplier $\Lambda \in L^{2}((0, T) ; \mathbb{R})$ is such that

$$
\frac{d}{d t} \int \chi=0
$$

For the Allen-Cahn equation (10), one can prove similar results as the ones above. Let us briefly recall the setting in the general case. The surface tensions are then given by

$$
\sigma_{i j}=d_{W}\left(\alpha_{i}, \alpha_{j}\right),
$$

where in general $d_{W}(u, v)$ denotes the geodesic distance in the state space $\mathbb{R}^{N}$ w.r.t. the metric $\sqrt{2 W(u)}\langle\cdot, \cdot$,$\rangle .$ For the sake of simplicity, the result is only stated in the simplest case of $N=1$ and the standard choice for the potential

$$
W(u)=\frac{1}{4}\left(1-u^{2}\right)^{2},
$$

which leads to the simpler equation (1) discussed in the introduction. Then the limit of the Cahn-Hilliard energy is a multiple of the perimeter, i.e.,

$$
F_{\varepsilon} \rightarrow \sigma \int|\nabla \chi|
$$

in the sense of $\Gamma$-convergence, cf. [30], where the surface tension is given by $\sigma=\int_{-1}^{1} \sqrt{2 W(s)} d s=\frac{2 \sqrt{2}}{3}$. The main result is the following convergence result which, as the other works discussed here, is conditional in the sense that we assume

$$
\int_{0}^{T} F_{\varepsilon}\left(u_{\varepsilon}\right) d t \rightarrow \sigma \int_{0}^{T} \int|\nabla \chi| d t .
$$

The result applies in the general context discussed in the introduction with mild conditions on the growth and convexity of the multi-well potential $W$ at $\infty$, cf. [21, (3)-(5)] 
Theorem 2.6. Let $T<\infty$ be an arbitrary finite time horizon. Given a sequence of well-prepared initial data $u_{\varepsilon, 0}:[0, \Lambda)^{d} \rightarrow \mathbb{R}$ in the sense that

$$
u_{\varepsilon, 0} \rightarrow 2\left(\chi_{0}-1\right) \text { a.e. and } \sigma \int\left|\nabla \chi_{0}\right|=\lim _{\varepsilon \downarrow 0} E_{\varepsilon}\left(u_{\varepsilon, 0}\right)<\infty,
$$

there exists a subsequence $\varepsilon \downarrow 0$ such that the solutions $u_{\varepsilon}$ of (10) with initial datum $u_{\varepsilon, 0}$ converge to $2(\chi-1)$ for some characteristic function $\chi \in L^{\infty}\left((0, T) ; B V\left([0, \Lambda)^{d} ;\{0,1\}\right)\right)$. If furthermore (23) holds, then $\chi$ moves by mean curvature according to Definition 2.3.

\section{Idea of proof}

Sketch of proof of Proposition 2.1. The minimizing movements interpretation (12) yields the a priori estimate

$$
\sup _{0<t<T} E_{h}\left(\chi_{h}\right)+\int_{0}^{T} \sqrt{h} \int\left(G_{h / 2} \star \partial_{t}^{h} \chi_{h}\right)^{2} \leq E_{0},
$$

where $\partial_{t}^{h} \chi_{h}(t):=\frac{\chi_{h}(t+h)-\chi_{h}(t)}{h}$ denotes the difference quotient with increment $h$. While the bound on the energy amounts to the following control on the modulus of continuity in space

$$
\int_{0}^{T} \int\left|\chi_{h}(x+v, t)-\chi_{h}(x, t)\right| \lesssim(1+T) E_{0}(|v|+\sqrt{h})
$$

for all $v \in \mathbb{R}^{d}$, cf. [19, Lemma 2.4]. This is almost a uniform BV-bound, which only fails on scales below the length scale $\sqrt{h}$.

Clearly, any uniform bound on the modulus of continuity in time below the time scale $h$ is hopeless. Even down to scales $h$ it is not obvious, as the estimate on the $L^{2}$ estimate on $\partial_{t}^{h} G_{h / 2}{ }^{\star} \chi_{h}$ is weighted by the degenerating factor $\sqrt{h} \downarrow 0$. Using the identity

$$
|\chi-\tilde{\chi}|=(\chi-\tilde{\chi})^{2}=(\chi-\tilde{\chi}) G_{h}{ }^{\star}(\chi-\tilde{\chi})+(\chi-\tilde{\chi})+\left(\chi-G_{h}{ }^{\star} \chi-\left(\tilde{\chi}-G_{h}{ }^{\star} \tilde{\chi}\right)\right),
$$

for $\chi=\chi(t+\tau)$ and $\tilde{\chi}=\chi(t)$ with $\tau \gtrsim \sqrt{h}$ together with the a priori bound and Jensen's inequality one obtains the estimate

$$
\int_{0}^{T} \int\left|\chi_{h}(x, t+\tau)-\chi_{h}(x, t)\right| \lesssim(1+T) E_{0}(\tau+\sqrt{h}),
$$

cf. [19, Lemma 2.5]. Combining the two bounds yields the compactness of $\chi_{h}$ in $L^{1}\left([0, \Lambda)^{d} \times(0, T)\right)$. Furthermore, one can easily post-process the bound (26) to a Hölder-type bounds in time with values in $L^{1}$ (which again fail on scales below $\sqrt{h}$ ), cf. [19, Lemma 2.6] and obtain that the limit $\chi$ is not only in the space $L^{\infty}\left((0, T) ; B V\left([0, \Lambda)^{d}\right)\right)$ but also in $C^{1 / 2}\left((0, T) ; L^{1}\left([0, \Lambda)^{d}\right)\right)$.

Sketch of proof of Theorem 2.2. The basic idea is to pass to the limit $h \rightarrow 0$ in the Euler-Lagrange equation of the minimizing movements interpretation (12). The Euler-Lagrange equation is obtained from taking inner variations of $\chi$, i.e.,

$$
\partial_{s} \chi_{s}+\xi \cdot \nabla \chi_{s}=0 .
$$

Using the smoothness of the test vector field, to leading order as $h \rightarrow 0$, one obtains the equation

$$
\int_{0}^{T} \int \xi \cdot \sqrt{h} \nabla G_{h}{ }^{\star} \chi_{h} \partial_{t}^{h} \chi_{h}=-\int_{0}^{T} \int \nabla \xi: \frac{1}{\sqrt{h}}\left(1-\chi_{h}\right)\left(G_{h} I d-h \nabla^{2} G_{h}\right){ }^{\star} \chi_{h} .
$$


The right-hand side converges:

$$
\lim _{h \rightarrow 0} \int_{0}^{T} \int \nabla \xi: \frac{1}{\sqrt{h}}\left(1-\chi_{h}\right)\left(G_{h} I d-h \nabla^{2} G_{h}\right) \star \chi_{h}=c_{0} \int_{0}^{T} \int \nabla \xi:(I d-v \otimes v)|\nabla \chi|,
$$

cf. [19, Proposition 3.1]. Indeed, the convergence of the curvature-term is similar to a classical statement of Reshetnyak [33] for the perimeter functional. The proof in the case of the thresholding energy is based on ideas from the $\Gamma$-convergence proof [10], in particular generalizing the consistency (13) to anisotropic (and tensor-valued) measures, and a generalization of the monotonicity (14), which however does not seem to be essential for the proof.

Let us turn to the main difficulty of the proof of Theorem 2.2, namely the convergence of the velocity-term

$$
\int_{0}^{T} \int \xi \cdot \sqrt{h} \nabla G_{h}{ }^{\star} \chi_{h} \partial_{t}^{h} \chi_{h}
$$

The structure of this term is

$$
\sqrt{h} \nabla G_{h}{ }^{\star} \chi_{h} \approx c_{0} v, \quad \partial_{t}^{h} \chi_{h} \rightarrow \partial_{t} \chi=V|\nabla \chi|,
$$

where a priori both convergences are weak.

Thinking of the test vector field $\xi$ as localizing on a small space-time patch, one is lead to "freezing " the approximate normal $\sqrt{h} \nabla G_{h}{ }^{\star} \chi^{h}$, i.e., replacing it by $c_{0} v^{\star}$, where $v^{\star} \in S^{d-1}$ is a constant unit vector. This is possible at the expense of an error measured in terms of a combination of the time derivative the local energy gap, that is, the energy excess, which (modulo a localization) is of the form

$$
E_{h}\left(\chi_{h}\right)-E_{h}\left(\chi^{*}\right) \text { where } \chi^{*} \text { is a half space in direction } v^{*} .
$$

In fact, this step is the heart of the paper [19] and has to be carried out on the mesoscopic time scale $\alpha \sqrt{h}$ instead of the microscopic time scale $h$ (where an additional fudge factor $\alpha>0$ comes into play). The interested reader is referred to the detailed outline of this part of the proof [19, pp. 35-38].

By the convergences (23) and (13), even when localizing with a partition of unity, this term converges to

$$
c_{0} \int|\nabla \chi|-c_{0} \int\left|\nabla \chi^{\star}\right| \text {. }
$$

After replacing the approximate normal $\sqrt{h} \nabla G_{h}{ }^{\star} \chi_{h}$, one is left with the convergent term

$$
c_{0} \int_{0}^{T} \int \xi \cdot v^{\star} \partial_{t}^{h} \chi_{h} \rightarrow c_{0} \int_{0}^{T} \int \xi \cdot v^{\star} \partial_{t} \chi=c_{0} \int_{0}^{T} \int \xi \cdot v^{\star} V|\nabla \chi| .
$$

After the limit $h \rightarrow 0$, one can "unfreeze" the normal at the expense of an error which is a combination of the tilt excess

$$
\int_{0}^{T} \int\left|v-v^{\star}\right|^{2}|\nabla \chi|
$$

and the $L^{2}$-norm of the normal velocity, which is bounded. Hence, passing first to the limit $h \rightarrow 0$, then taking the fineness of the localization to zero and finally the fudge factor $\alpha \rightarrow 0$ one obtains the convergence

$$
\lim _{h \rightarrow 0} \int_{0}^{T} \int \xi \cdot \sqrt{h} \nabla G_{h}{ }^{\star} \chi_{h} \partial_{t}^{h} \chi_{h}=c_{0} \int \xi \cdot v V|\nabla \chi| .
$$


Sketch of proof of Theorem 2.4. First, observe that the basic a priori estimate (25) is not sharp. Indeed, the prefactor of the metric term in (25) is off by the factor $\frac{1}{2}$. De Giorgi's ideas to overcome this issue in his general framework was to find a variational interpolation of the time discretizations which in case of thresholding is given by

$$
u_{h}((n+\lambda) h) \in \arg \min \left\{\frac{1}{\sqrt{h}} \int(1-u) G_{h} * u+\frac{1}{\lambda \sqrt{h}} \int\left[G_{h / 2} \star\left(u-\chi_{n}\right)\right]^{2}\right\},
$$

where $n \in \mathbb{N}$ and $\lambda \in(0,1]$ and the minimum runs over all measurable $u:[0, \Lambda)^{d} \rightarrow \mathbb{R}$ with $0 \leq u \leq 1$ a.e. Applying the abstract theory of minimizing movements in metric spaces [3, Chapter 3] to thresholding leads to an evolution equation for the energy along this interpolation which yields the energy-dissipation equality

$$
E_{h}\left(\chi_{h}\left(t_{2}\right)\right)+\int_{t_{1}}^{t_{2}} \sqrt{h} \int\left(G_{h / 2} \star \partial_{t}^{h} \chi_{h}\right)^{2}+\int_{t_{1}}^{t_{2}} \sqrt{h} \int\left(G_{h / 2} \star\left(\frac{u_{h}(t)-\chi_{h}(t)}{h}\right)\right)^{2}=E_{h}\left(\chi_{h}\left(t_{1}\right)\right) .
$$

which yields the sharp inequality

$$
\int\left|\nabla \chi\left(t_{2}\right)\right|+\int_{t_{1}}^{t_{2}} \int H^{2}|\nabla \chi| \leq \int\left|\nabla \chi\left(t_{1}\right)\right|
$$

in the limit $h \rightarrow 0$, cf. [20, Proposition 4.8].

This is however only one global inequality, whereas Brakke's weak formulation comes as a family of localized inequalities. The basis of the analysis in [20] is the following simple but rather important observation which is particular about the thresholding scheme and is for example not known for the scheme [2]: Thresholding does not only solve one global minimization problem but a whole family of localized problems. Indeed, for any non-negative test function $\zeta$, the iterate $\chi_{n}$ obtained from Algorithm 0.1 minimizes the functional

$$
\begin{aligned}
& \frac{1}{\sqrt{h}} \int \zeta(1-u) G_{h}{ }^{\star} u+\frac{1}{\sqrt{h}} \int\left(u-\chi_{n-1}\right)\left[\zeta, G_{h}{ }^{\star}\right]\left(1-\chi_{n-1}\right) \\
& +\frac{1}{\sqrt{h}} \int\left(u-\chi_{n-1}\right)\left[\zeta, G_{h / 2}{ }^{\star}\right] G_{h / 2}{ }^{\star}\left(u-\chi_{n-1}\right)+\frac{1}{\sqrt{h}} \int \zeta\left[G_{h / 2}{ }^{\star}\left(u-\chi_{n-1}\right)\right]^{2}
\end{aligned}
$$

among all $u$ such that $0 \leq u \leq 1$, where

$$
\left[\zeta, G_{h}^{\star}\right] u:=\zeta G_{h}{ }^{\star} u-G_{h}{ }^{\star}(\zeta u) \approx 2 \sqrt{h} \nabla \zeta \cdot \sqrt{h} \nabla G_{h}{ }^{\star} u
$$

denotes the commutator of the multiplication with the test function $\zeta$ and the convolution with the kernel $G_{h}$.

Repeating the same argument as above with the penalization $\frac{1}{\lambda}$ only in front of the localized metric term, i.e., the last term in (28), one obtains a localized energy equality, where now the interpolation $u_{h}$ depends on the positive test function $\zeta$ as well.

The contribution of the commutator in the metric term, i.e., the third term in (28) is negligible while the second term yields the transport term in Brakke’s inequality, cf. [20, Proposition 4.5].

Sketch of proof of Theorem 2.5. It turns out, cf. [22, Lemma 1.7], that the scheme of Ruuth and Wetton is equivalent to the same minimization problem (12) subject to the volume constraint $\int \chi_{n}=\int \chi_{n-1}$, which may also be expressed by the Lagrange multiplier $\frac{2 \lambda_{n}-1}{\sqrt{h}}$.

The additional difficulty compared to Theorems 2.1 and 2.2 is that one needs an estimate on the threshold values $\lambda_{n}$.

The natural $L^{2}$-estimate on the Lagrange multiplier $\langle H\rangle$, which is available for a solution of volumepreserving mean-curvature flow (20) by Jensen's inequality, and (21) corresponds precisely to the necessary estimate on the (piecewise constant interpolation of the) threshold value $\lambda_{h}=\lambda_{h}(t)$ of the form $\left|\lambda_{h}-\frac{1}{2}\right|=$ $O(\sqrt{h})$ in $L^{2}(0, T)$, i.e.,

$$
\sup _{h>0} \int_{0}^{T}\left(\frac{2 \lambda_{h}-1}{\sqrt{h}}\right)^{2}<\infty .
$$


This estimate can be obtained from the Euler-Lagrange equation of the minimizing movements interpretation with an additional Lagrange multiplier $\frac{2 \lambda_{n}-1}{\sqrt{h}}$. Indeed, the problem boils down to finding a test vector field $\xi$ such that $\nabla \xi$ is uniformly bounded while $\int(\nabla \cdot \xi) \chi_{n}$ is bounded below. Such a vector field can be achieved as the gradient of the solution of a simple elliptic problem, [22, Proposition 1.12].

This estimate on the Lagrange multiplier and Chebyshev's inequality imply furthermore that for all but finitely many iterations one has $\left|\lambda_{n}-\frac{1}{2}\right|<\frac{1}{4}$, which in particular allows to prove the tightness in the sense that the evolution stays in a bounded region, cf. [22, Lemmas 1.15 and 1.16].

Sketch of proof of Theorem 2.6. On the one hand, the analysis of the Allen-Cahn equation is simpler in the sense that one does not have to analyze the non-continuous thresholding operator and a multi-scale analysis as in $[19,22]$ is not necessary. The compactness part of the theorem has been basically solved (except for minor refinement to the multi-phase case).

On the other hand, a new difficulty arises, namely the richer geometry of the state space. To illustrate this difficulty, let us imagine to be far away from triple junctions at an almost flat part of the limit interface $\Sigma_{i j}$. Along the normal direction the solutions $u_{\varepsilon}$ roughly performs a transition between the two wells $\alpha_{i}, \alpha_{j} \in \mathbb{R}^{N}$ and (away from multiple junctions) this transition is expected to follow geodesics (w.r.t. the metric $\sqrt{2 W(u)}(\cdot, \cdot)$ ) between these two wells. As these geodesics are in general not straight lines (not even unique in general), it is clear that even under the assumption that the energies converge, the gradients $\nabla u_{\varepsilon}$ do not converge strongly. In the best case, the gradients would behave like rank-one matrices $\delta u_{\varepsilon} \otimes v_{\varepsilon}$ such that $\delta u_{\varepsilon} \in \mathbb{R}^{N}$ is tangential to the geodesic (and therefore oscillates as $\varepsilon \rightarrow 0$ ) while $v_{\varepsilon} \approx v^{\star} \in \mathbb{R}^{d}$ is almost constant close to the limiting interface.

In fact one can prove that, given the convergence of the energies, the following approximate tilt-excess

$$
\int \frac{1}{\varepsilon}\left|\varepsilon \nabla u_{\varepsilon}-\partial_{u} \phi_{i}\left(u_{\varepsilon}\right) \otimes v^{\star}\right|^{2}
$$

is controlled by (a multi-phase version of) the tilt-excess of the limit [21, Lemma 3.4]. Here $\phi_{i}(u):=d_{W}\left(u, \alpha_{i}\right)$, so that $\partial_{u} \phi_{i}\left(u_{\varepsilon}\right) \in \mathbb{R}^{N}$ corresponds to $\delta u_{\varepsilon}$ mentioned above. In the simpler two-phase case, $\phi(u)=$ $\int_{-1}^{u} \sqrt{2 W(s)} d s$ so that $\partial_{u} \phi\left(u_{\varepsilon}\right)=\sqrt{2 W\left(u_{\varepsilon}\right)}$.

The main idea to recover the distributional solution for the limit is to test the Allen-Cahn equation (1) with $\xi \cdot \varepsilon \nabla u_{\varepsilon}$, which yields

$$
\int_{0}^{T} \int \xi \cdot \varepsilon \nabla u_{\varepsilon} \partial_{t} u_{\varepsilon}=\int_{0}^{T} \int\left(\varepsilon \Delta u_{\varepsilon}-\frac{1}{\varepsilon} W^{\prime}\left(u_{\varepsilon}\right)\right) \xi \cdot \nabla u_{\varepsilon} .
$$

While the convergence of the left-hand side is a result of Luckhaus and Modica [23] in the two-phase case and can be generalized to the multi-phase case by quantifying their Reshetnyak-type argument [21, Proposition 3.1], the main difficulty is the convergence of the velocity-term, i.e., the left-hand side. However, the approximate tilt-excess (29) allows to freeze the approximate normal $\varepsilon \nabla u_{\varepsilon}$ in the sense that one may replace it by $\sqrt{2 W\left(u_{\varepsilon}\right)} v^{\star}$ and the chain rule yields the convergence of the velocity-term, cf. [21, Proposition 3.5].

\section{Open problems}

1. Generalize these methods to smooth anisotropies and to Neumann or Dirichlet boundary conditions.

2. Does the Voronoi Implicit Interface Method [35] dissipate any energy?

3. Find an unconditional convergence result for two-phase thresholding to a general Brakke flow. The main difficulty here is similar to the equipartition of energy in [16].

4. A rather difficult open problem is the generalization of Ilmanen's proof [16] to the multi-phase case.

5. The strong convergence can be proven in special cases, as for example for the thresholding scheme for short time [37]. Does the condition hold for a regular network? Does it hold for mean convex (i.e., $H \geq 0$ ) initial conditions? 
6. Any weak-strong or generic uniqueness statement for multi-phase mean-curvature flow would be desirable.

\section{References}

[1] Samuel M. Allen and John W. Cahn. A microscopic theory for antiphase boundary motion and its application to antiphase domain coarsening. Acta Metallurgica, 27(6):1085-1095, 1979.

[2] Fred Almgren, Jean E. Taylor, and Lihe Wang. Curvature-driven flows: a variational approach. SIAM Journal on Control and Optimization, 31(2):387-438, 1993.

[3] Luigi Ambrosio, Nicola Gigli, and Giuseppe Savaré. Gradient flows in metric spaces and in the space of probability measures. Birkhäuser, 2008.

[4] Sisto Baldo. Minimal interface criterion for phase transitions in mixtures of Cahn-Hilliard fluids. In Annales de l'IHP Analyse non linéaire, volume 7, pages 67-90, 1990.

[5] Guy Barles and Christine Georgelin. A simple proof of convergence for an approximation scheme for computing motions by mean curvature. SIAM Journal on Numerical Analysis, 32(2):484-500, 1995.

[6] Antonin Chambolle. An algorithm for mean curvature motion. Interfaces and Free Boundaries, (6):195-218, 2004.

[7] Yun G. Chen, Yoshikazu Giga, and Shun'ichi Goto. Uniqueness and existence of viscosity solutions of generalized mean curvature flow equations. Journal of Differential Geometry, 33(3):749-786, 1991.

[8] Ennio De Giorgi. New problems on minimizing movements. Boundary Value Problems for PDE and Applications, 29:91-98, 1993.

[9] Selim Esedoğlu. Motion of grain boundaries in polycrystalline materials. http://www.math.lsa.umich.edu/ esedoglu/ Research/grains/grains.html, 2016.

[10] Selim Esedoğlu and Felix Otto. Threshold dynamics for networks with arbitrary surface tensions. Communications on Pure and Applied Mathematics, 68(5):808-864, 2015.

[11] Lawrence C. Evans. Convergence of an algorithm for mean curvature motion. Indiana University Mathematics Journal, 42(2):533-557, 1993

[12] Lawrence C. Evans, H. Mete Soner, and Panagiotis E. Souganidis. Phase transitions and generalized motion by mean curvature. Communications on Pure and Applied Mathematics, 45(9):1097-1123, 1992.

[13] Lawrence C. Evans and Joel Spruck. Motion of level sets by mean curvature I. Journal of Differential Geometry, 33(3):635-681, 1991.

[14] Lawrence C. Evans and Joel Spruck. Motion of level sets by mean curvature IV. The Journal of Geometric Analysis, 5(1):77-114, 1995.

[15] Gerhard Huisken. Asymptotic behavior for singularities of the mean-curvature flow. Journal of Differential Geometry, 31(1):285-299, 1990

[16] Tom Ilmanen. Convergence of the Allen-Cahn equation to Brakkes motion by mean curvature. Journal of Differential Geometry, 38(2):417-461, 1993.

[17] Tom Ilmanen, André Neves, and Felix Schulze. On short time existence for the planar network flow. arXiv preprint arXiv:1407.4756, 2014

[18] Lami Kim and Yoshihiro Tonegawa. On the mean curvature flow of grain boundaries. Annales de l'Institut Fourier (Grenoble), 66(1):43-142, 2017.

[19] Tim Laux and Felix Otto. Convergence of the thresholding scheme for multi-phase mean-curvature flow. Calculus of Variations and Partial Differential Equations, 55(5):1-74, 2016.

[20] Tim Laux and Felix Otto. Brakke's inequality for the thresholding scheme, arXiv preprint arXiv:1708.03071

[21] Tim Laux and Thilo Simon. Convergence of the Allen-Cahn equation to multi-phase mean-curvature flow. arXiv preprint arXiv:1606.07318, 2016

[22] Tim Laux and Drew Swartz. Convergence of thresholding schemes incorporating bulk effects. Interfaces and Free Boundaries, (19):273-304, 2017

[23] Stephan Luckhaus and Luciano Modica. The Gibbs-Thompson relation within the gradient theory of phase transitions. Archive for Rational Mechanics and Analysis, 107(1):71-83, 1989.

[24] Stephan Luckhaus and Thomas Sturzenhecker. Implicit time discretization for the mean curvature flow equation. Calculus of Variations and Partial Differential Equations, 3(2):253-271, 1995.

[25] Carlo Mantegazza, Matteo Novaga, and Alessandra Pluda. Motion by curvature of networks with two triple junctions. arXiv preprint arXiv:1606.08011, 2016.

[26] Carlo Mantegazza, Matteo Novaga, and Vincenzo Maria Tortorelli. Motion by curvature of planar networks. Annali della Scuola Normale Superiore di Pisa. Classe di Scienze. Serie V, 3(2):235-324, 2004.

[27] Barry Merriman, James K. Bence, and Stanley J. Osher. Diffusion generated motion by mean curvature. CAM Report 92-18, 1992. Department of Mathematics, University of California, Los Angeles. 
[28] Barry Merriman, James K. Bence, and Stanley J. Osher. Motion of multiple junctions: A level set approach. Journal of Computational Physics, 112(2):334-363, 1994.

[29] Peter W. Michor and David Mumford. Riemannian geometries on spaces of plane curves. Journal of the European Mathematical Society, 8(1):1-48, 2006.

[30] Luciano Modica and Stefano Mortola. Un esempio di Gamma-convergenza. Bolletino della Unione Matematica Itialana $B$ (5), 14(1):285-299, 1977.

[31] William W. Mullins. Two-dimensional motion of idealized grain boundaries. Journal of Applied Physics, 27(8):900-904, 1956.

[32] William T. Read and William B. Shockley. Dislocation models of crystal grain boundaries. Physical Review, 78(3):275, 1950.

[33] Yu G. Reshetnyak. Weak convergence of completely additive vector functions on a set. Siberian Mathematical Journal, 9(6):1039-1045, 1968.

[34] Steven J. Ruuth and Brian T. R. Wetton. A simple scheme for volume-preserving motion by mean curvature. Journal of Scientific Computing, 19(1-3):373-384, 2003.

[35] Robert I. Saye and James A. Sethian. The voronoi implicit interface method for computing multiphase physics. Proceedings of the National Academy of Sciences, 108(49):19498-19503, 2011.

[36] Oliver Schnürer, Abderrahim Azouani, Marc Georgi, Juliette Hell, Nihar Jangle, Amos Koeller, Tobias Marxen, Sandra Ritthaler, Mariel Sáez, and Felix Schulze. Evolution of convex lens-shaped networks under the curve shortening flow. Transactions of the American Mathematical Society, 363(5):2265-2294, 2011.

[37] Drew Swartz and Nung Kwan Yip. Convergence of diffusion generated motion to motion by mean curvature. Communications in Partial Differential Equations, 42(10), 1598-1643, 2017. 